\title{
NEUTRON STAR CORES IN THE GENERAL RELATIVISTIC THOMAS-FERMI TREATMENT
}

\author{
RICCARDO BELVEDERE* ${ }^{*}$ JORGE A. RUEDA ${ }^{\dagger}$ and REMO RUFFINI ${ }^{\ddagger}$ \\ Physics Department, Sapienza - University of Rome \\ and \\ ICRA - International Center for Relativistic Astrophysics \\ P. le Aldo Moro 5 - Rome, Italy 00185 \\ ICRANet \\ Piazza della Repubblica 10 - Pescara, Italy 65122 \\ *riccardo.belvedere@icra.it \\ †jorge.rueda@icra.it \\ ${ }^{\ddagger}$ ruffini@icra.it
}

\begin{abstract}
We introduce a new set of equations to describe the equilibrium of the core of neutron stars, composed by self-gravitating degenerate neutrons, protons and electrons in $\beta$-equilibrium. We take into account strong, weak, electromagnetic and gravitational interactions within the framework of general relativity. We extend the conditions of equilibrium based on the constancy of the Klein potentials to the strongly interactive case. The strong interactions between nucleons are modeled through the exchange of the $\sigma, \omega$ and $\rho$ virtual mesons. The equations are solved numerically in the case of zero temperatures and for a non-rotating spherically symmetric neutron stars in the mean-field approximation.
\end{abstract}

\section{Introduction}

In a series of recent articles, ${ }^{1-3}$ the firsts steps to a new consistent treatment for the description of neutron stars, taking into account the strong, weak, electromagnetic and gravitational interactions, have been developed. First of all, it has been generalized the Feynman, Metropolis and Teller treatment of compressed atoms to the relativistic regimes, ${ }^{1}$ including the condition for nuclear equilibrium. This approach has been extrapolated to compressed nuclear matter cores of stellar dimensions with mass numbers $A \approx\left(m_{\text {Planck }} / m_{n}\right)^{3} \sim 10^{57}$, where $m_{\text {Planck }}$ is the Planck's mass and $m_{n}$ is the neutron rest-mass, thanks to the existence of scaling laws of the relativistic Thomas-Fermi equation in the ultrarelativistic regime. Such configurations fulfill global but not local charge neutrality and they have electric fields on the core surface, reaching values much larger than the critical value $E_{c}=m_{e}^{2} c^{3} /(e \hbar)$. In a recent work by Rotondo et al., in Ref. 2, the effects of gravitation have been introduced by considering the simplest case of a general relativistic system of degenerate fermions composed of neutrons, protons and electrons in $\beta$-equilibrium, neglecting the strong interactions between nucleons. There, it has been shown that the local 
charge neutrality violates necessary conditions of equilibrium at the microphysical scale, and has been proved the existence of a solution with global charge neutrality taking into account gravito-electrodynamical effects, i.e. gravito-polarization.

Here we show, based on recent progress, ${ }^{3}$ how the previous treatment is generalized to the case of strong interactions. In addiction, we integrate the new set of equations of equilibrium in the mean-field approximation for neutron star cores. The matching of these solutions to the crust of the neutron star will be presented in a forthcoming publication.

\section{The Constitutive Equations}

We assume here neutron star cores as composed by a system of degenerate neutrons, protons and electrons in $\beta$-equilibrium. The strong interactions between nucleons are modeled in a Yukawa-like fashion by the exchange of the $\sigma, \omega$ and $\rho$ virtual mesons: ${ }^{4,5} \sigma$ is an isoscalar meson field providing the attractive long-range part of the nuclear force; $\omega$ is a massive vector field that models the repulsive short range nuclear force; $\rho$ is the massive isovector field that takes account surface and isospin effects of the nuclei, modeling a repulsive nuclear force. ${ }^{6,7}$

The total Lagrangian density of the system has to take into account gravitational, strong, weak and electromagnetic interactions and is given by

$$
\begin{aligned}
\mathcal{L}=\mathcal{L}_{g}+\mathcal{L}_{f}+\mathcal{L}_{\sigma}+\mathcal{L}_{\omega}+\mathcal{L}_{\rho}+\mathcal{L}_{\gamma}+\mathcal{L}_{\text {int }} \\
\mathcal{L}_{g}=-\frac{R}{16 \pi G} \\
\mathcal{L}_{\gamma}=-\frac{1}{4} F_{\mu \nu} F^{\mu \nu} \\
\mathcal{L}_{\sigma}=\frac{1}{2} \nabla_{\mu} \sigma \nabla^{\mu} \sigma-U(\sigma) \\
\mathcal{L}_{\omega}=-\frac{1}{4} \Omega_{\mu \nu} \Omega^{\mu \nu}+\frac{1}{2} m_{\omega}^{2} \omega_{\mu} \omega^{\mu} \\
\mathcal{L}_{\rho}=-\frac{1}{4} \mathcal{R}_{\mu \nu} \mathcal{R}^{\mu \nu}+\frac{1}{2} m_{\rho}^{2} \rho_{\mu} \rho^{\mu} \\
\mathcal{L}_{f}=\sum_{i=e, N} \bar{\psi}_{i}\left(i \gamma^{\mu} D_{\mu}-m_{i}\right) \psi_{i}, \\
\mathcal{L}_{\text {int }}=-g_{\sigma} \sigma \bar{\psi}_{N} \psi_{N}-g_{\omega} \omega_{\mu} J_{\omega}^{\mu}-g_{\rho} \rho_{\mu} J_{\rho}^{\mu}+e A_{\mu} J_{\gamma, e}^{\mu}-e A_{\mu} J_{\gamma, N}^{\mu},
\end{aligned}
$$

where $\mathcal{L}_{g}, \mathcal{L}_{\gamma}, \mathcal{L}_{\sigma}, \mathcal{L}_{\omega}$ and $\mathcal{L}_{\rho}$ are the Lagrangian densities for the free-fields, $\mathcal{L}_{f}$ is the Lagrangian density for the three fermion species and $\mathcal{L}_{\text {int }}$ is the interacting part of the Lagrangian density. $\Omega_{\mu \nu} \equiv \partial_{\mu} \omega_{\nu}-\partial_{\nu} \omega_{\mu}, \mathcal{R}_{\mu \nu} \equiv \partial_{\mu} \rho_{\nu}-\partial_{\nu} \rho_{\mu}, F_{\mu \nu} \equiv$ $\partial_{\mu} A_{\nu}-\partial_{\nu} A_{\mu}$ are the field strength tensors for the $\omega^{\mu}, \rho^{\mu}$ and $A^{\mu}$ fields respectively, $\nabla_{\mu}$ stands for covariant derivative and $R$ is the Ricci scalar. We adopt the Lorenz gauge for the fields $A_{\mu}, \omega_{\mu}$, and $\rho_{\mu}$. The self-interaction scalar field potential $U(\sigma)$ is a quartic-order polynom for a renormalizable theory (see e.g. Ref. 8). The conserved 
currents are

$$
\begin{aligned}
J_{\omega}^{\mu} & =\bar{\psi}_{N} \gamma^{\mu} \psi_{N}, \\
J_{\rho}^{\mu} & =\bar{\psi}_{N} \tau_{3} \gamma^{\mu} \psi_{N}, \\
J_{\gamma, e}^{\mu} & =\bar{\psi}_{e} \gamma^{\mu} \psi_{e}, \\
J_{\gamma, N}^{\mu} & =\bar{\psi}_{N}\left(\frac{1+\tau_{3}}{2}\right) \gamma^{\mu} \psi_{N},
\end{aligned}
$$

where $g_{\sigma}, g_{\omega}$ and $g_{\rho}$ are the coupling constant of the $\sigma, \omega$ and $\rho$-fields respectively, and $e$ is the fundamental electric charge. The Dirac matrices $\gamma^{\mu}$ and the isospin Pauli matrices satisfy the Dirac algebra in curved spacetime (see e.g. Ref. 9).

The equations of motion of the above Lagrangian lead to the Einstein-MaxwellDirac system of equations is given

$$
\begin{aligned}
G_{\mu \nu}+8 \pi G T_{\mu \nu} & =0, \\
\nabla_{\mu} F^{\mu \nu}-e J_{c h}^{\nu} & =0, \\
\nabla_{\mu} \Omega^{\mu \nu}+m_{\omega}^{2} \omega^{\nu}-g_{\omega} J_{\omega}^{\nu} & =0, \\
\nabla_{\mu} \mathcal{R}^{\mu \nu}+m_{\rho}^{2} \rho^{\nu}-g_{\rho} J_{\rho}^{\nu} & =0, \\
\nabla_{\mu} \nabla^{\mu} \sigma+\partial_{\sigma} U(\sigma)+g_{s} n_{s} & =0, \\
{\left[\gamma_{\mu}\left(i D^{\mu}-V_{N}^{\mu}\right)-\tilde{m}_{N}\right] \psi_{N} } & =0, \\
{\left[\gamma_{\mu}\left(i D^{\mu}+e A^{\mu}\right)-m_{e}\right] \psi_{e} } & =0,
\end{aligned}
$$

where the scalar density is $n_{s}=\bar{\psi}_{N} \psi_{N}$, the nucleon effective mass is $\tilde{m}_{N} \equiv m_{N}+$ $g_{\sigma} \sigma$, and

$$
V_{N}^{\mu} \equiv g_{\omega} \omega^{\mu}+g_{\rho} \tau \rho^{\mu}+e\left(\frac{1+\tau_{3}}{2}\right) A^{\mu},
$$

is the effective four potential of nucleons. The energy-momentum tensor of free-fields and free-fermions $T^{\mu \nu}$ of the system (3)-(6) is

$$
T^{\mu \nu}=T_{f}^{\mu \nu}+T_{\gamma}^{\mu \nu}+T_{\sigma}^{\mu \nu}+T_{\omega}^{\mu \nu}+T_{\rho}^{\mu \nu},
$$

where

$$
\begin{aligned}
& T_{\gamma}^{\mu \nu}=F_{\alpha}^{\mu} F^{\alpha \nu}+\frac{1}{4} g^{\mu \nu} F_{\alpha \beta} F^{\alpha \beta}, \\
& T_{\sigma}^{\mu \nu}=\nabla^{\mu} \nabla^{\nu} \sigma-g^{\mu \nu}\left[\frac{1}{2} \nabla_{\sigma} \sigma \nabla^{\sigma} \sigma-U(\sigma)\right] \\
& T_{\omega}^{\mu \nu}=\Omega_{\alpha}^{\mu} \Omega^{\alpha \nu}+\frac{1}{4} g^{\mu \nu} \Omega_{\alpha \beta} \Omega^{\alpha \beta}+m_{\omega}^{2}\left(\omega^{\mu} \omega^{\nu}-\frac{1}{2} g^{\mu \nu} \omega_{\alpha} \omega^{\alpha}\right), \\
& T_{\rho}^{\mu \nu}=\mathcal{R}_{\alpha}^{\mu} \mathcal{R}^{\alpha \nu}+\frac{1}{4} g^{\mu \nu} \mathcal{R}_{\alpha \beta} \mathcal{R}^{\alpha \beta}+m_{\rho}^{2}\left(\mathcal{R}^{\mu} \mathcal{R}^{\nu}-\frac{1}{2} g^{\mu \nu} \mathcal{R}_{\alpha} \omega^{\alpha}\right),
\end{aligned}
$$

are the contribution due to free-fields and $T_{f}^{\mu \nu}$ is the contribution of free-fermions

$$
T_{f}^{\mu \nu}=(\mathcal{E}+\mathcal{P}) u^{\mu} u^{\nu}-\mathcal{P} g^{\mu \nu},
$$


where $u^{\mu}$ is the four-velocity of the fluid which satisfies $u^{\mu} u_{\mu}=1$, and the energydensity $\mathcal{E}$ and the pressure $\mathcal{P}$ are given by

$$
\mathcal{E}=\sum_{i=n, p, e} \mathcal{E}_{i}, \quad \mathcal{P}=\sum_{i=n, p, e} \mathcal{P}_{i},
$$

being $\mathcal{E}_{i}$ and $\mathcal{P}_{i}$ the single fermion fluid contributions

$$
\begin{aligned}
\mathcal{E}_{i} & =\frac{2}{(2 \pi)^{3}} \int_{0}^{P_{i}^{F}} \epsilon_{i}(p) 4 \pi p^{2} d p, \\
\mathcal{P}_{i} & =\frac{1}{3} \frac{2}{(2 \pi)^{3}} \int_{0}^{P_{i}^{F}} \frac{p^{2}}{\epsilon_{i}(p)} 4 \pi p^{2} d p,
\end{aligned}
$$

where $p$ is the momentum of the particle, $\epsilon_{i}(p)=\sqrt{p^{2}+\tilde{m}_{i}^{2}}$ denote the singleparticle energy-spectrum and $P_{i}^{F}$ is the Fermi momentum of the $i$-specie.

\section{Equations for the Core}

We consider non-rotating spherically symmetric neutron stars, so we introduce the spacetime metric

$$
d s^{2}=\mathrm{e}^{\nu(r)} d t^{2}-\mathrm{e}^{\lambda(r)} d r^{2}-r^{2} d \theta^{2}-r^{2} \sin ^{2} \theta d \varphi^{2},
$$

Due the huge number of particles involved in the core of a neutron star, of the order of $10^{57}$, it is possible adopt the mean-field approximation, in which fermion-field operators are replaced by their expectation values (see e.g. Ref. 5). Moreover, in the static case, only the time-components of the currents survive, so that, taking the expectation values of Eqs (9)-(12) we obtain

$$
\begin{gathered}
J_{0}^{c h}=n_{c h} u_{0}=\left(n_{p}-n_{e}\right) u_{0}, \\
J_{0}^{\omega}=n_{b} u_{0}=\left(n_{n}+n_{p}\right) u_{0}, \\
J_{0}^{\rho}=n_{3} u_{0}=\left(n_{p}-n_{n}\right) u_{0},
\end{gathered}
$$

where $n_{b}, n_{p}, n_{n}$ and $n_{e}$ are the baryon, proton, neutron and electron number densities which are functions only of the spatial coordinates, and $u_{0}=\sqrt{g_{00}}=e^{\nu / 2}$. Under these conditions, the system of equations (13)-(19) becomes ${ }^{3}$

$$
\begin{aligned}
e^{-\lambda(r)}\left(\frac{1}{r^{2}}-\frac{\lambda^{\prime}}{r}\right)-\frac{1}{r^{2}} & =-8 \pi G T_{0}^{0}, \\
e^{-\lambda(r)}\left(\frac{1}{r^{2}}+\frac{\nu^{\prime}}{r}\right)-\frac{1}{r^{2}} & =-8 \pi G T_{1}^{1}, \\
\mathcal{P}^{\prime}+\frac{\nu^{\prime}}{2}(\mathcal{E}+\mathcal{P}) & =-g_{\sigma} n_{s} \sigma^{\prime}-\omega^{\prime} g_{\omega} J_{\omega}^{0}-\rho^{\prime} g_{\rho} J_{\rho}^{0}-V^{\prime} e J_{c h}^{0}, \\
V^{\prime \prime}+V^{\prime}\left[\frac{2}{r}-\frac{\left(\nu^{\prime}+\lambda^{\prime}\right)}{2}\right] & =-e^{\nu+\lambda} e J_{c h}^{0},
\end{aligned}
$$




$$
\begin{aligned}
& \sigma^{\prime \prime}+\sigma^{\prime}\left[\frac{2}{r}+\frac{\left(\nu^{\prime}-\lambda^{\prime}\right)}{2}\right]=e^{\nu+\lambda}\left[\partial_{\sigma} U(\sigma)+g_{s} n_{s}\right], \\
& \omega^{\prime \prime}+\omega^{\prime}\left[\frac{2}{r}-\frac{\left(\nu^{\prime}+\lambda^{\prime}\right)}{2}\right]=-e^{\nu+\lambda}\left[g_{\omega} J_{\omega}^{0}-m_{\omega}^{2} \omega^{0}\right], \\
& \rho^{\prime \prime}+\rho^{\prime}\left[\frac{2}{r}-\frac{\left(\nu^{\prime}+\lambda^{\prime}\right)}{2}\right]=-e^{\nu+\lambda}\left[g_{\rho} J_{\rho}^{0}-m_{\rho}^{2} \rho^{0}\right],
\end{aligned}
$$

where $A_{0} \equiv V$.

\section{Generalized Fermi Energies (Klein Potentials) and $\beta$-Equilibrium}

The nucleon doublet and the electronic spinor written in the phase-space are

$$
\psi_{i}=\psi_{i}(k) e^{-\imath k_{\mu} x^{\mu}},
$$

where $k$ denotes the " $k$ "-state and $k^{\mu}$ the corresponding wave number of the fermion specie.

From the Dirac equations for the nucleons and the electrons, we obtain

$$
\left(\gamma_{\mu} \mathcal{K}^{\mu}-\tilde{m}_{i}\right) \psi_{i}(k)=0
$$

with $\mathcal{K}^{\mu} \equiv k^{\mu}-V_{i}^{\mu}, V_{e}=-e V$. Making a quadrature of the Dirac operators in the phase-space we obtain the Fermi energy (the generalized chemical potentials or, for short, Klein potentials) for neutrons $E_{n}^{F}$, for protons $E_{p}^{F}$, and for electrons $E_{e}^{F}$

$$
\begin{aligned}
& E_{n}^{F}=\sqrt{g_{00}} \mu_{n}+g_{\omega} \omega-g_{\rho} \rho, \\
& E_{p}^{F}=\sqrt{g_{00}} \mu_{p}+g_{\omega} \omega+g_{\rho} \rho+e V, \\
& E_{e}^{F}=\sqrt{g_{00}} \mu_{e}-e V=e^{\nu / 2} \mu_{e}-e V .
\end{aligned}
$$

\section{Constancy of the Klein Potentials}

Using the equations of motion for the meson-fields $\sigma, \omega$, and $\rho$, and using the generalized Gibbs-Duhem relation ${ }^{3}$

$$
d \mathcal{P}=\sum_{i=n, p, e} n_{i} d \mu_{i}-g_{\sigma} n_{s} d \sigma,
$$

the energy-momentum conservation equation (36) can be rewritten as

$$
\sum_{i=n, p, e} n_{i} d\left(e^{\nu / 2} \mu_{i}\right)+g_{\omega} n_{B} d \omega+g_{\rho} n_{3} d \rho+e n_{c h} d V=0 .
$$

Using Eqs. (43)-(45), the Eq. (47) becomes

$$
\sum_{i=n, p, e} n_{i} d E_{i}^{F}=0,
$$


from which we obtain for independent and non-zero particle densities $n_{i}$, the constancy of the Klein Potentials

$$
\begin{aligned}
E_{e}^{F} & =e^{\nu / 2} \mu_{e}-e V=\text { constant } \\
E_{n, p}^{F} & =e^{\nu / 2} \mu_{n, p}+\mathcal{V}_{n, p}=\text { constant }
\end{aligned}
$$

where $\mathcal{V}_{n, p} \equiv g_{\omega} \omega+g_{\rho} \tau \rho+e\left(\frac{1+\tau_{3}}{2}\right) V$. The constants $E_{i}$ are linked by the condition of $\beta$-equilibrium of the nuclear matter

$$
E_{n}^{F}=E_{p}^{F}+E_{e}^{F} .
$$

\section{Integration of the Core Equations}

Through the new relations obtained in Secs. (4) and (5), the system of equation for the core can be rewritten in the form

$$
\begin{aligned}
e^{-\lambda(r)}\left(\frac{1}{r^{2}}-\frac{\lambda^{\prime}}{r}\right)-\frac{1}{r^{2}} & =-8 \pi G T_{0}^{0}, \\
e^{-\lambda(r)}\left(\frac{1}{r^{2}}+\frac{\nu^{\prime}}{r}\right)-\frac{1}{r^{2}} & =-8 \pi G T_{1}^{1}, \\
V^{\prime \prime}+V^{\prime}\left[\frac{2}{r}-\frac{\left(\nu^{\prime}+\lambda^{\prime}\right)}{2}\right] & =-e^{\nu+\lambda} e J_{c h}^{0}, \\
\partial_{\sigma} U(\sigma)+g_{s} n_{s} & =0, \\
g_{\omega} J_{\omega}^{0}-m_{\omega}^{2} \omega^{0} & =0, \\
g_{\rho} J_{\rho}^{0}-m_{\rho}^{2} \rho^{0} & =0, \\
E_{e}^{F}=e^{\nu / 2} \mu_{e}-e V & =\text { constant }, \\
E_{p}^{F}=e^{\nu / 2} \mu_{p}+e V+C_{\omega} n_{B}+C_{\rho} n_{3} & =\text { constant }, \\
E_{n}^{F}=E_{p}^{F}+E_{e}^{F} &
\end{aligned}
$$

where we have replaced the energy-momentum conservation equation with appropriate conservation laws for the Klein potentials and the $\beta$-equilibrium condition, and we have, as usual, approximated the massive fields as locally uniform ${ }^{5}$ (homogeneous in space) $C_{i}=\left(g_{i} / m_{i}\right)^{2}$.

In order to integrate the equilibrium equations we need to fix the parameters of the nuclear model, namely, fixing the coupling constants $g_{s}, g_{\omega}$ and $g_{\rho}$, and the meson masses $m_{\sigma}, m_{\omega}$ and $m_{\rho}$. Conventionally, such constants are fixed by fitting experimental properties of nuclei. Usual experimental properties of ordinary nuclei include saturation density, binding energy per nucleon (or experimental masses), symmetry energy, surface energy, and nuclear incompressibility. In Table 1 we present the following selected fits of the nuclear parameters: NL3, ${ }^{10} \mathrm{NL}-\mathrm{SH},{ }^{11}$ TM1, ${ }^{12}$ and TM2. ${ }^{13}$

In Figs. 1 and 2 are shown the results of the integration of equations (52)-(60), using the values of the parameters shown in Table $1 .{ }^{14}$ 
Table 1. Selected parameter sets of the $\sigma-\omega-\rho$ model.

\begin{tabular}{ccccc}
\hline & NL3 & NL-SH & TM1 & TM2 \\
\hline$m_{\sigma}(\mathrm{MeV})$ & 508.194 & 526.059 & 511.198 & 526.443 \\
$m_{\omega}(\mathrm{MeV})$ & 782.501 & 783.000 & 783.000 & 783.000 \\
$m_{\rho}(\mathrm{MeV})$ & 763.000 & 763.000 & 770.000 & 770.000 \\
$g_{s}$ & 10.2170 & 10.4440 & 10.0289 & 11.4694 \\
$g_{\omega}$ & 12.8680 & 12.9450 & 12.6139 & 14.6377 \\
$g_{\rho}$ & 4.4740 & 4.3830 & 4.6322 & 4.6783 \\
$g_{2}\left(\mathrm{fm}^{-1}\right)$ & -10.4310 & -6.9099 & -7.2325 & -4.4440 \\
$g_{3}$ & -28.8850 & -15.8337 & 0.6183 & 4.6076 \\
$c_{3}$ & 0.0000 & 0.0000 & 71.3075 & 84.5318 \\
\hline
\end{tabular}
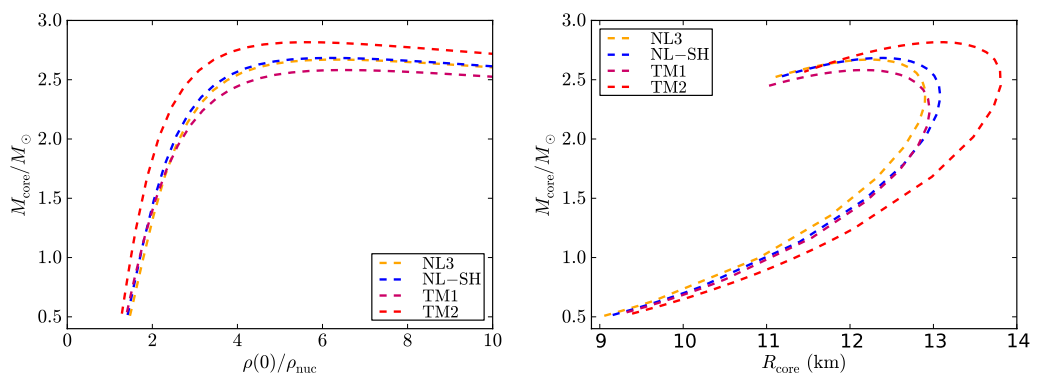

Fig. 1. Mass-central density (left panel) and mass-radius relations (right panel) for the core of neutron stars, calculated through the parameters set shown in Table 1.
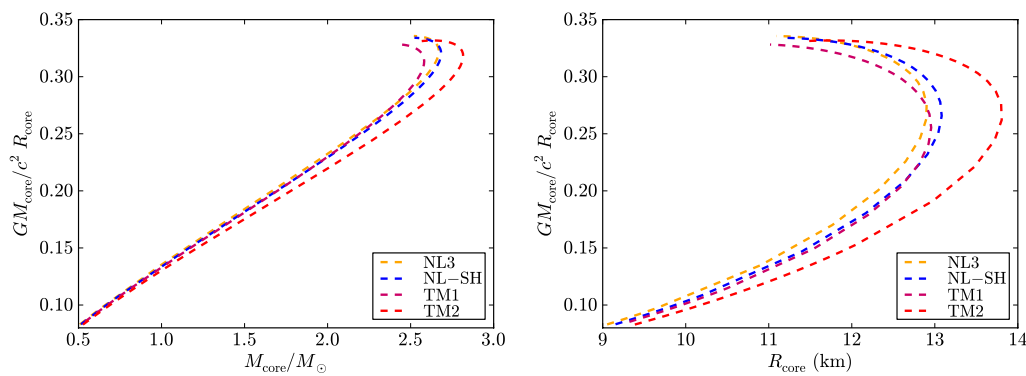

Fig. 2. Compactness-mass (left panel) and compactness-radius (right panel) for the core of neutron stars, calculated through the parameters set shown in Table 1.

These neutron star cores are also characterized by the presence of an electric field due to the charge separation induced by the cation of gravity on the different electron and proton rest masses, i.e. gravito-polarization. The Coulomb potential depth is of order $V \sim m_{\pi} c^{2} / e$, where $m_{\pi}$ is the pion rest mass. The electric field is $E / E_{c} \sim\left(m_{p} / m_{\text {Planck }}\right)\left(m_{\pi} / m_{e}\right)^{2} \sim 10^{-14}$, where $E_{c}=m_{e}^{2} c^{3} /(e \hbar)$ is the critical field for vacuum polarization. The neutron star cores presented here need to be 
properly matched to the crust of the star taking into due account the constancy of the Klein potentials at the core-crust interface. ${ }^{14}$

\section{References}

1. M. Rotondo, Jorge A. Rueda, R. Ruffini and S.-S. Xue, Phys. Rev. C 83, 045805 (2011).

2. M. Rotondo, Jorge A. Rueda, R. Ruffini and S.-S. Xue, Phys. Lett. B 701, 667 (2011).

3. J. A. Rueda, R. Ruffini and S.-S. Xue, Nucl. Phys. A 872, 286 (2011).

4. H. P. Duerr, Phys. Rev. D 103, 469 (1956).

5. J. D. Walecka Annals Phys. 83, 491 (1974).

6. J. Boguta and A. R. Bodmer, Nucl. Phys. A 292, 413 (1977).

7. P. Ring, Prog. Part. Nucl. Phys. 37, 193 (1996).

8. T. D. Lee and G. C. Wick, Phys. Rev. D 9, 2291 (1974).

9. T. D. Lee and Y. Pang, Phys. Rev. D 35, 3678 (1987).

10. G. A. Lalazissis, J. König, and P. Ring, Phys. Rev. C 55, 540 (1997).

11. M. M. Sharma, M. A. Nagarajan, and P. Ring, Phys. Lett. B 312, 337 (1993).

12. Y. Sugahara and H. Toki, Nucl. Phys. A 579, 557 (1994).

13. D. Hirata, H. Toki, and I. Tanihata, Nucl. Phys. A 589, 239 (1995).

14. R. Belvedere, D. Pugliese, Jorge A. Rueda, R. Ruffini and S.-S. Xue, accepted in Nucl. Phys. A. 